\title{
Tunable field-induced superconductivity
}

\author{
W. Gillijns, A. V. Silhanek, and V. V. Moshchalkov \\ INPAC-Institute for Nanoscale Physics and Chemistry, K.U. Leuven, Celestijnenlaan 200D, B-3001 Leuven, Belgium
}

(Received 4 September 2006; published 14 December 2006)

\begin{abstract}
We investigate the transport properties of a thin superconducting Al layer covering a square array of magnetic dots with out-of-plane magnetization. A thorough characterization of the magnetic properties of the dots allowed us to fine-tune their magnetic state at will, hereby changing the influence of the dots on the superconductor in a continuous way. We show that even though the number of vortex-antivortex pairs discretely increases with increasing the magnetization of the dots, no corresponding discontinuity is observed in the resistance of the sample. The evolution of the superconducting phase boundary as the magnetic state of the dots is swept permits one to devise a fully controllable and erasable field-induced superconductor.
\end{abstract}

DOI: 10.1103/PhysRevB.74.220509

PACS number(s): 74.78.-w, 74.25.Dw

The hallmark of superconductivity and the technological applications based on it is the possibility to carry electrical currents without resistance below the critical temperature $T_{c}$. This nondissipative quantum state is however suppressed either by applying a magnetic field or by submitting the system to a high-enough electrical current as to break the Cooper pairs responsible for the superconducting condensate. In type-II superconductors which are most interesting for practical applications, an applied magnetic field $H\left(H_{c 1}<H\right.$ $<H_{c 2}$, with $H_{c 1}$ and $H_{c 2}$ the lower and upper critical fields) penetrates the superconductor in the form of flux quanta. Under the influence of an applied current these vortices start moving, hereby destroying the nondissipative state. During the last decade enormous efforts have been devoted to prevent this drawback by anchoring vortices with different types of pinning potentials. Particular attention has been focused on magnetic pinning centra originally due to their promising enhancement of the critical current. ${ }^{1}$ Interestingly, it was also found that in such superconductor/ferromagnet $(S / F)$ hybrid systems field compensation effects between an applied magnetic field and the stray fields of the ferromagnets can drastically change the superconducting properties. ${ }^{2,3}$

It has recently been shown that if an array of out-of-plane magnetized dots is deposited on top of a superconducting film, the $T_{c}(H)$ phase boundary can be shifted by exactly an integer number of flux quanta per unit cell. ${ }^{4,5}$ The occurrence of a maximum in $T_{c}$ at a nonzero magnetic field results from the compensation of the dots' field by the applied field. The reason for the quantized character of field-induced superconductivity (FIS) lies in the ability of the superconductor to quantize the flux generated by a magnetic dot, whatever its value, by either compensating the field excess or generating the field deficiency with supercurrents. If the dots generate a small flux, supercurrents will counterbalance this flux and no compensation effects are present. However, if the flux crosses a certain critical value the field lines generated by the dot penetrate the superconductor in the form of vortexantivortex (VAV) pair(s) and hence and the maximum critical temperature will shift by exactly an integer number of flux quanta per unit cell. This picture suggests that for particular magnetization values the field locus of the maximum $T_{c}$ should undergo abrupt displacements from $n \phi_{0}$ to $(n+1) \phi_{0}$ with $n$ integer. So far, the detailed evolution of this shift by an integer number of flux quanta has remained unveiled, mostly due to the difficulties to control the magnetization of the dots in a continuous fashion. Here we explore the evolution of the superconducting properties of a thin-film superconductor, now deposited on top of an array of tunable magnetic dots.

The sample under investigation is a superconducting $\mathrm{Al}$ film of thickness $d=50 \mathrm{~nm}$ evaporated on top of a square array of magnetic dots with $2 \mu \mathrm{m}$ lattice constant. A $5 \mathrm{~nm}$ thick Si buffer layer was first evaporated on top of the dots to avoid proximity effects. In this way the interaction between the ferromagnet and the superconductor is purely electromagnetic in origin. An atomic force microscopy picture of the dots' array is shown in Fig. 1. The ferromagnetic dots have a diameter of $1.36 \mu \mathrm{m}$ and consist of a $2.5 \mathrm{~nm}$ Pt buffer layer covered with a $[0.4 \mathrm{~nm} \mathrm{Co} / 1.0 \mathrm{~nm} \mathrm{Pt}]_{10}$ multilayer with magnetization perpendicular to the sample surface. ${ }^{6}$

The magnetic properties of the dots were investigated using a commercial Quantum Design superconducting quan-

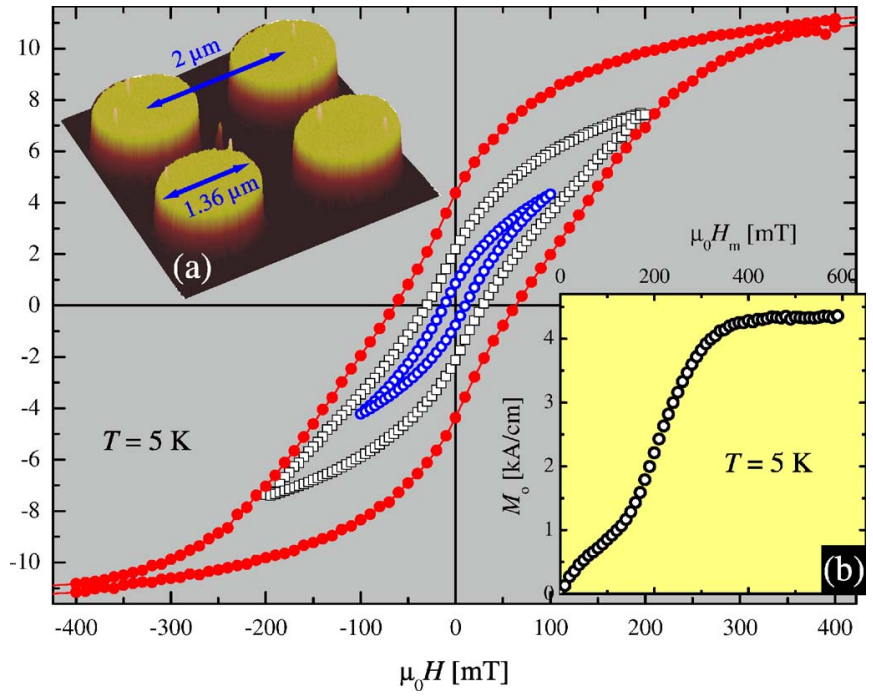

FIG. 1. (Color online) Magnetization loops of the Co/Pt dots for three different excursion fields measured at $5 \mathrm{~K}$. The insets show (a) an atomic force microscopy picture of the magnetic dots and (b) the remanent magnetization $M_{0}$ as a function of the maximum applied field $H_{m}$ starting from the demagnetized state $\mu_{0} H_{m}=0 \mathrm{mT}$. 


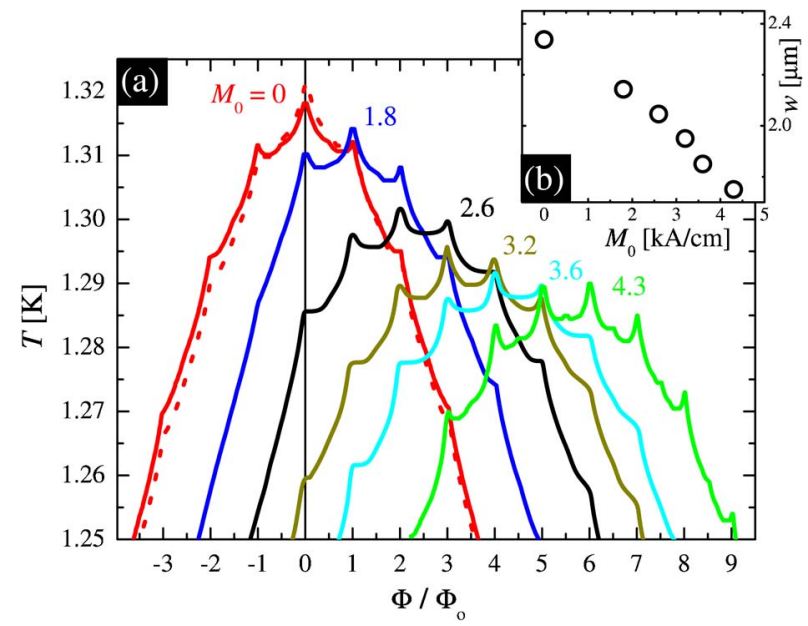

FIG. 2. (Color online) (a) Superconducting transition $T_{c}(H)$ of the Al film for different magnetic states of the dots. By increasing the magnetization a clear shift of $T_{c}(H)$ and a decrease of $T_{c}^{\max }$ is observed. (b) Lateral dimension $w$ of the nucleation of superconductivity as a function of the magnetization of the dots.

tum interference device (SQUID) magnetometer. The main panel of Fig. 1 presents the field dependence of the magnetization $M(H)$ for the $\mathrm{Co} / \mathrm{Pt}$ dots at $T=5 \mathrm{~K}$, where the magnetization was determined by using the total volume of the dot. The broader magnetization loop (filled circles) was recorded after saturation in a field of $\mu_{0} H_{s}=1 \mathrm{~T}$. After following a careful demagnetization procedure it is possible to reduce the remanent magnetization down to $0.5 \%$ of the saturation value. As a consequence of the large diameter of the dots, the demagnetized state microscopically corresponds to a magnetic multidomain state with very little stray field. ${ }^{4}$ Starting from the demagnetized state minor loops can be built up by making field excursions $H_{m}$ with $H_{m}<H_{s}$ (open symbols). Every minor loop has a unique remanent magnetization value $M_{0}$ associated with it. Repeating this procedure for several $H_{m}$ we determined $M_{0}$ as a function of $H_{m}$ as shown in Fig. 1(b). It is important to note that the full control of the out-of-plane magnetization of the dots relies on the one-to-one correlation between $M_{0}$ and $H_{m}$ together with the reproducibility of the minor loops.

From the superconducting phase boundary for the demagnetized dots we determine a critical temperature $T_{c}$ of $1.343 \mathrm{~K}$ and a superconducting coherence length $\xi(0)$ $=117 \mathrm{~nm}$. Using the electronic mean free path $l \approx 15 \pm 1 \mathrm{~nm} \ll \xi(0)$ as estimated from the normal state resistance above $T_{c}$ we determined a penetration depth $\lambda(0)$ $\sim 100 \mathrm{~nm}$ in the dirty limit. ${ }^{8}$ The effective penetration depth due to the thin-film geometry is $\Lambda=\lambda^{2} / d$, which gives $200 \mathrm{~nm}$ for our sample. From this value we obtained a Ginzburg-Landau parameter $\kappa=\Lambda / \xi \approx 1.7$ showing that our sample is a type-II superconductor.

Let us first analyze the evolution of the superconducting phase boundary $T_{c}(H)$ as the magnetization of the dots is gradually increased. Figure 2(a) shows a selected set of these data determined by a $10 \%$ normal-state resistance criterion. As expected, when the dots are in the demagnetized state $\left(M_{0}=0\right)$ a symmetric $T_{c}(H)$ boundary is observed. It is worth noticing that this boundary nearly reproduces that corresponding to the virgin state (dotted line) as a consequence of having a similar multidomain state of the dots. As the outof-plane magnetization of the dots crosses some critical value, a vortex is created on top of the dot while the corresponding antivortex is located in between the dots. The presence of this vortex antivortex pair (VAV) shifts $T_{c}^{\max }$ to $\phi$ $=\phi_{0}$. The reason for this shift is that an external applied field $\phi=\phi_{0}$ will introduce an extra vortex per unit cell which in turn annihilates the interstitial antivortex. Since the effective field between the dots is minimal for this particular field a maximum $T_{c}$ is obtained in this case. Further increase of the magnetization of the dots results in a shift of the phase boundary by an integer number $n$ of quantum flux units $\phi_{0}$ and in a decrease of the maximum $T_{c}^{\max }$. This decrease of $T_{c}^{\max }$ is a result of the increasing average field felt by the superconductor. It is clear that by controlling the magnetic states of the dots it is possible to place the position of $T_{c}^{\max }$ at any desired $n<7$. The maximum shift is ultimately determined by the maximal flux generated by the dots. This value can be increased by either increasing the dot size or by increasing the saturation magnetization.

For all $M_{0}$ values and sufficiently high temperatures it can be seen that $T_{c}(H)$ exhibits a parabolic background. A similar behavior has been reported for $F / S$ bilayers ${ }^{9}$ and samples with square arrays of antidots ${ }^{10}$ and can be attributed to the change of dimensionally when $\xi(T)$ exceeds the width $w$ of the areas where superconductivity first nucleates. Within this regime the phase boundary can be approximated by ${ }^{11}$ $T_{c}(H) / T_{c}(0)=1-(\alpha H)^{2}$, with $\alpha=\xi(0) \pi w / 2 \sqrt{3} \phi_{0}$. By using this expression to fit the data in Fig. 2(a) we estimate $w$ as a function of $M_{0}$ [as shown in Fig. 2(b)]. Here a continuous decrease of $w$ with $M_{0}$ is observed which is consistent with the reduction of the available nucleation area due to the increasing strength of the magnetic template created by the dots.

The previous description of the evolution of $T_{c}(H)$ with increasing $M_{0}$ would remain incomplete without determining whether the transition from $n$ to $n+1$ VAV pairs actually occurs as a sudden horizontal displacement. In order to address this issue we monitored the evolution of the resistance $R(H)$ at a nearly constant reduced temperature $t=0.991$ as the magnetization of the dots $M_{0}$ is increased. This procedure turns out to be far more sensitive than just following the evolution of the $T_{c}(H)$ phase boundary itself. In Fig. 3(a) we present these measurements for the particular case of $n=1$, although the complete spectrum of accessible magnetization values was experimentally spanned. ${ }^{12}$ For the sake of clarity the curves in Fig. 3(a) have been displaced horizontally along the field axis.

The leftmost curve exhibits a very symmetric shape with a minimum resistance centered at $\phi=\phi_{0}$ and two local dips at $\phi=0$ and $\phi=2 \phi_{0}$. This particular magnetization value corresponds to the generation of exactly one VAV pair. As $M_{0}$ is increased the central dip slowly moves upward whereas the satellite dip at $\phi=2 \phi_{0}$ becomes deeper. Strikingly, at a certain critical magnetization $M_{c 2}$ both local minima reach the same level. From that point on the absolute minimum resistance is located at $\phi=2 \phi_{0}$ and eventually a new symmetric 

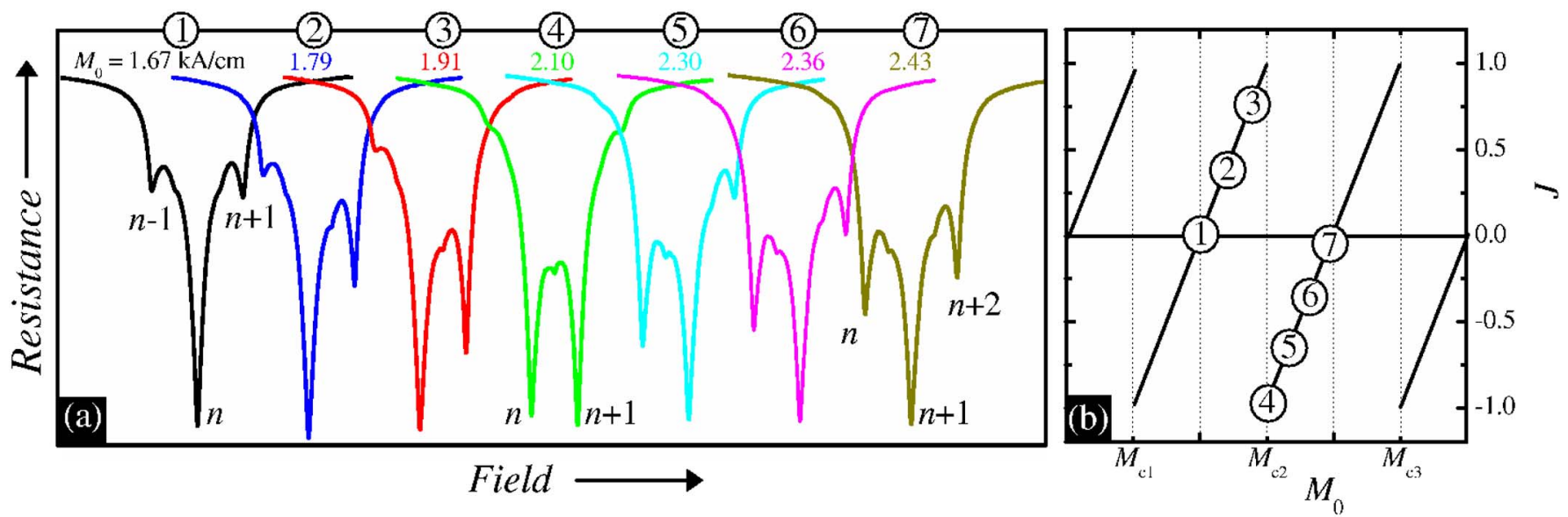

FIG. 3. (Color online) (a) Magnetoresistance measurements for different values of the magnetization $M_{0}$ of the magnetic dots at a constant reduced temperature $t=T / T_{c}$. The presented data correspond to the transition of the location of the maximum $T_{c}$ from $\phi=\phi_{0}$ to $2 \phi_{0}$. For clarity the curves have been displaced horizontally. (b) Illustration of the supercurrents encircling the magnetic dots as a function of the magnetization. Colored symbols correspond to the colored curves in panel (a). Starting from the black curve, the increase of the magnetization will result in increasing currents, counteracting the field of the dots and keeping the flux exactly equal to $\phi_{0}$. Crossing a critical value $M_{c 2}$ the currents reverse polarity hereby generating an extra vortex-antivortex pair. Further increasing the magnetization will result in a decrease of the currents since the flux is increasingly carried by the dot itself.

configuration centered at $\phi=2 \phi_{0}$ is obtained. The above described evolution shows that even though the absolute minimum of the $R(H)$ jumps from $\phi=\phi_{0}$ to $\phi=2 \phi_{0}$ at $M=M_{c 2}$, at any field the resistance remains a continuous function of $M_{0}$. A similar evolution is observed for switching from $n$ to $n$ +1 with $n<7 .^{12}$

In order to explain the above described behavior it is crucial to take the screening supercurrents $J$ into consideration, very much like in the Little-Parks effect ${ }^{14}$ [see Fig. 3(b)]. For $M_{0}=1.67 \mathrm{kA} / \mathrm{cm}$, at $\phi=\phi_{0}$ one flux line is located at the magnetic dot and no total currents are present since the flux of the vortex is provided by the field of the dot [1 in Fig. $3(b)]$. Under these circumstances both vortices and antivortices feel the same interaction with the dot and thus the satellite dips at $\phi=0$ and $\phi=2 \phi_{0}$ are equally deep. Upon increasing the magnetization, the excess of field generated by the dot is counteracted by the supercurrents [(2) and (3) in Fig. $3(b)$. These currents break the previous symmetry favoring the presence of vortices over antivortices thus accounting for the deepening of the minimum at $\phi=2 \phi_{0}$ and the rising of the minimum at $\phi=0$. This unbalanced situation persists up to a certain magnetization $M_{c 2}$. Crossing this value [4 in Fig. $3(b)]$ results in the creation of an extra VAV pair, thus shifting the phase boundary to $\phi=2 \phi_{0}$. At the same time the currents circling the dots have reversed polarity, now favoring antivortices rather than vortices. This interaction is reflected in the reversal of the asymmetry with respect to the new minimum. Further increasing $M_{0}$ reduces the asymmetry as the current progressively approaches zero [5 and (6) in Fig. 3(b)]. Eventually a fully symmetric curve centered at $\phi=2 \phi_{0}$ is recovered [7 in Fig. 3(b)].

It is worth emphasizing that although the local minima in the resistance move up and down in a continuous manner as $M_{0}$ is swept, the field position of the absolute minimum of the $R(H)$ curves always undergoes a discrete jump every time a critical value $M_{c}$ is crossed. This situation becomes more evident in Fig. 4 where a contour plot of $R\left(H, M_{0}\right)$ at $t=0.991$ is shown. The dark islands in this graph indicate the location of the lower resistance. A clear stepwise structure associated with the discrete increase of VAV pairs as $M_{0}$

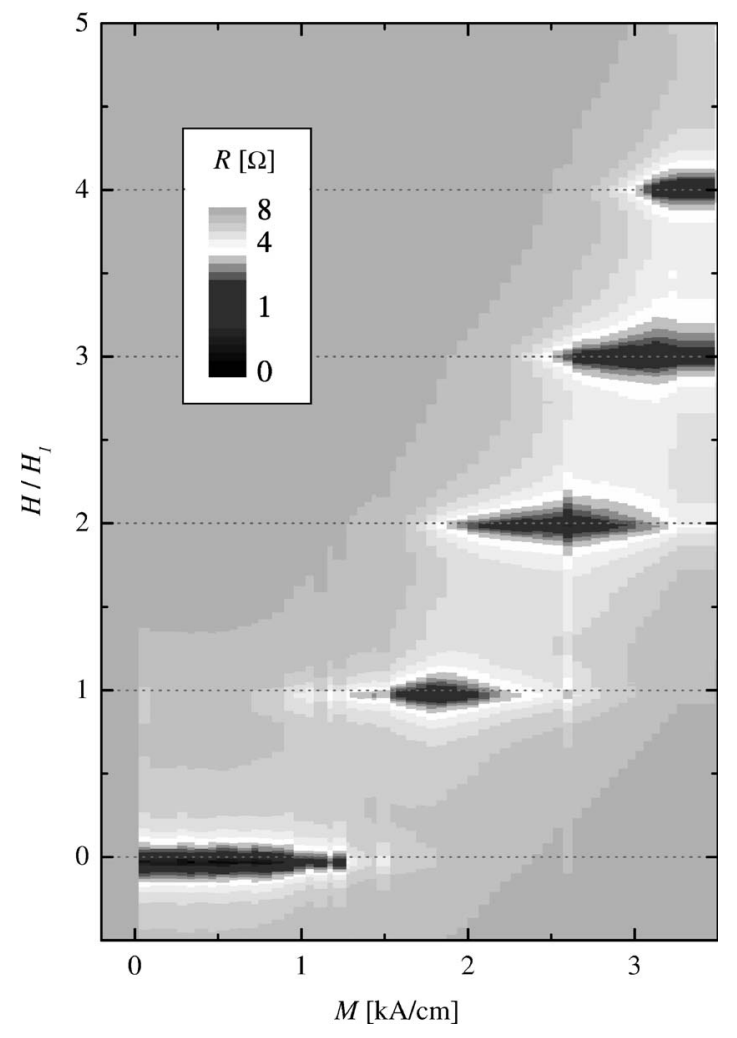

FIG. 4. Contour plot of the resistance as a function of field and magnetization for $t=0.991$. Dark islands correspond to lowest resistance and hence highest critical temperature. The steplike profile is a consequence of the quantization of the magnetic field in an integer number of flux quanta by the superconductor. 
rises, can be discerned. From this plot one can accurately determine the magnetization needed to generate the first, second, third, and fourth VAV pairs.

It is interesting to note that the generation of the first vortex-antivortex pair appears delayed with respect to the subsequent steps. This finding is consistent with previous theoretical predictions by Milosevic and Peeters ${ }^{5}$ based on the solution of the Ginzburg-Landau equations. In that work it is shown that for an analogous hybrid system with similar superconducting and magnetic properties to ours the induction of the first VAV pair needs a larger flux than for the following transitions. The ultimate reason for this effect lies in the higher degree of symmetry of the $n=0 \mathrm{VAV}$ state with respect to the $n \neq 0$ states. Our results represent an experimental confirmation of this prediction. In a later report the same authors ${ }^{13}$ have predicted that the $T_{c}\left(M_{0}\right)$ phase boundary corresponding to an array of tunable magnetic dots should exhibit cusplike features associated with to the generation of VAV pairs superimposed on a global decrease of $T_{c}$ with increasing $M_{0}$. This global reduction of $T_{c}$ is clearly visible in Fig. 2(a). And since the critical temperature is determined by the lowest resistance, the crossing of the two minimal dips in the $R(H)$ curves at $M_{c}$ will result in a cusplike feature in the $T_{c}\left(M_{0}\right)$ phase boundary as well.

As a last remark, we would like to point out that the observed similarities with the Little-Parks ${ }^{14}$ effect should not be pushed too far. Although in both cases the currents adjust themselves to quantize the magnetic field, in our system the field is provided by the dots rather than by a homogeneous source giving rise to VAV pairs. Also, the spatial coexistence of screening and vortex currents is not present in our system since vortices can move around in the two-dimensional lattice.

In conclusion, we have shown that the remanent magnetization $M_{0}$ of out-of-plane magnetized dots can be tuned from nearly zero (demagnetized) to a material-dependent maximum value. This continuous degree of freedom allowed us to observe and investigate tunable field-induced superconductivity and the progressive evolution of the superconducting phase boundary as a function of $M_{0}$ and thus reveal the microscopic mechanisms that lead to discrete jumps in $T_{c}^{\max }$ without having discontinuities in the resistance at any point. The remarkable flexibility attainable with these magnetic field resistant superconductors makes them very attractive for practical applications.

This work was supported by the Fund for Scientific Research-Flanders FWO-Vlaanderen, the Belgian InterUniversity Attraction Poles IUAP, the Research Fund K.U. Leuven GOA/2004/02, and by the Japan-EU-USA Nanoscience and Engineering in Superconductivity (NES) programs. A.V.S. is grateful for the support from the FWOVlaanderen. We would also like to thank IMEC for making the resist patterns.
${ }^{1}$ L. N. Bulaevskii, E. M. Chudnovsky, and M. P. Maley, Appl. Phys. Lett. 76, 2594 (2000).

${ }^{2}$ A. I. Buzdin, Rev. Mod. Phys. 77, 935 (2005).

${ }^{3}$ I. F. Lyuksyutov and V. L. Pokrovsky, Adv. Phys. 54, 67 (2005).

${ }^{4}$ M. Lange, M. J. Van Bael, Y. Bruynseraede, and V. V. Moshchalkov, Phys. Rev. Lett. 90, 197006 (2003).

${ }^{5}$ M. V. Milosevic and F. M. Peeters, Phys. Rev. Lett. 93, 267006 (2004).

${ }^{6}$ W. B. Zeper et al., J. Appl. Phys. 65, 4971 (1989).

${ }^{7}$ It is worth noticing that the magnetization of the dots exhibit almost no temperature dependence for $5 \mathrm{~K}<T<300 \mathrm{~K}$. Due to the large separation between dots there is little interaction between them and the properties of the array mimic the properties of an individual dot. Although the present report deals with circular-shaped dots, both the tuneability and demagnetization procedure are independent of the actual geometry of the dots.
${ }^{8}$ M. Tinkham, Introduction to Superconductivity, 2nd ed. (McGraw-Hill, New York, 1996), p. 120.

${ }^{9}$ M. Lange, M. J. Van Bael, and V. V. Moshchalkov, Phys. Rev. B 68, 174522 (2003).

${ }^{10}$ E. Rosseel et al., Physica C 282-287, 1567 (1997).

${ }^{11}$ V. V. Moshchalkov et al., in Superconductivity in Networks and Mesoscopic Systems, edited by C. Giovanella and C. J. Lambert (AIP, New York, 1998), p. 171.

${ }^{12}$ See EPAPS Document No. E-PRBMDO-74-R12646 for a movie (Gillijns-etal.avi) showing the field dependence of the resistance as a function of the magnetization. For more information on EPAPS, see http://www.aip.org/pubservs/epaps.html

${ }^{13}$ M. V. Milosevic and F. M. Peeters, Phys. Rev. Lett. 94, 227001 (2005).

${ }^{14}$ W. A. Little and R. D. Parks, Phys. Rev. Lett. 9, 9 (1962). 